\title{
Confluence of Shallow Right-Linear Rewrite Systems ${ }^{\star}$
}

\author{
Guillem Godoy ${ }^{1}$ and Ashish Tiwari ${ }^{2}$ \\ 1 Technical University of Catalonia \\ Jordi Girona 1, Barcelona, Spain \\ ggodoy@lsi.upc.es \\ 2 SRI International, Menlo Park, CA 94025 \\ tiwari@csl.sri.com
}

\begin{abstract}
We show that confluence of shallow and right-linear term rewriting systems is decidable. This class of rewriting system is expressive enough to include nontrivial nonground rules such as commutativity, identity, and idempotence. Our proof uses the fact that this class of rewrite systems is known to be regularity-preserving, which implies that its reachability and joinability problems are decidable. The new decidability result is obtained by building upon our prior work for the class of ground term rewriting systems and shallow linear term rewriting systems. The proof relies on the concept of extracting more general rewrite derivations from a given rewrite derivation.
\end{abstract}

\section{Introduction}

Term rewriting systems provide a Turing-complete formalism for modeling computation. Terms over a signature encode the state of a system and the rewriting rules specify the dynamics. Rewriting systems have been used this way to model and verify discrete state transition systems, see for instance $[11,2,6]$. Under a slightly different interpretation, rewriting rules can be viewed as defining an equational theory over terms. The direction of the rule, in this case, generally indicates which equivalent form is simpler. This viewpoint has been successfully used for equational reasoning in theorem proving, see for instance [1].

Confluence is a central property of rewrite systems. It guarantees that the order of application of rewrite rules is not significant. When viewed as a model of computation, confluence provides a more general definition of determinism. For purposes of verification, confluence generalizes the condition required for partial-order reduction. In the context of equational reasoning, confluence and termination of a computable rewrite relation imply decidability of the word problem for the induced equational theory.

The expressive power of a rewrite system can be limited by imposing additional constraints on the form of terms. For instance, if variables are not allowed,

\footnotetext{
* The first author was partially supported by Spanish Min. of Educ. and Science by the LogicTools project (TIN2004-03382). The second author was supported in part by the National Science Foundation under grants ITR-CCR-0326540 and CCR-0311348.
} 
we get ground term rewrite system, which have been extensively studied, mainly via mapping them to tree automata [3]. Richer classes of rewrite systems are obtained by allowing restricted variable occurrences in the term rewrite system (or the tree automata transitions). In going from special to more general classes of rewrite systems, the complexity of deciding various fundamental problems, like termination and confluence, increases until all these problems become undecidable. It is, therefore, fruitful to study these properties for some intermediate classes, especially if they are expressive enough to capture interesting rules.

In this context, we consider shallow right-linear term rewrite systems, where every rule $l \rightarrow r$ is such that every variable occurs at most once in $r$, and all variables in $l, r$ occur at depth 0 or 1 . Some examples of shallow right-linear rules are $0 \wedge x \rightarrow 0, x \wedge x \rightarrow x, 1 \wedge x \rightarrow x, x \vee x \rightarrow x$ and $x \vee y \rightarrow y \vee x$

The class of shallow right-linear rewrite systems is very close to the frontier of classes for which confluence is undecidable. A (generally) simpler problem like reachability is known to be undecidable for linear TRS's, and also for shallow TRS's [10]. On the positive side, Takai, Kaji, and Seki [13] showed that right-linear finite-path-overlapping systems effectively preserve recognizability. Since shallow right-linear systems are right-linear and finite-path-overlapping, it follows that the reachability and joinability problems for these systems are decidable. The exact location of the barrier for decidability of termination and confluence inside the class of right-linear finite-path-overlapping systems is still open.

We prove the decidability of confluence for shallow right-linear TRS's. This result uses the decidability of reachability and joinability for this class as a black box. We extend and simplify the ideas presented in [8] where decidability of confluence of shallow linear TRS's was proved. Here, we eliminate the necessity of constructing a rewrite closure for the original TRS (which is difficult for shallow right-linear TRS's, if possible), and the notion of rewriting with marked (sub-)terms. Non-linearity forces us to use extended counterexample witnesses to confluence: pairs $\{s, t\}$ were used in [8], but now larger sets $\left\{s_{1}, \ldots, s_{n}\right\}$ are needed. Moreover, as in [8], the computation of top-stabilizable constants (constants equivalent to some term that cannot be reduced to a constant) is crucial, but much more difficult here. In fact, we can compute all such constants only when the system is confluent, and the final proof shows that when not all of them are computed, a non-confluence witness is detected.

The procedure to decide confluence of $R$ has two steps. First we add new rules to $R$ and obtain $\bar{R} \supseteq R$ in Section 3.1. Then, in Section 3.3, we present a simple decidable characterization of confluence of $R$ in terms of $R$ - and $\bar{R}$-joinability of certain flat terms.

\section{Preliminaries}

We use standard notation from the term rewriting literature. A signature $\Sigma$ is a (finite) set of function symbols, which is partitioned as $\cup_{i} \Sigma_{i}$ such that $f \in \Sigma_{n}$ if arity of $f$ is $n$. Symbols in $\Sigma_{0}$, called constants, are denoted by 
$a, b, c, d$, with possible subscripts. The elements of a set $\mathcal{V}$ of variable symbols are denoted by $x, y, z$ with possible subscripts. The set $\mathcal{T}(\Sigma, \mathcal{V})$ of terms over $\Sigma$ and $\mathcal{V}$, position $p$ in a term, subterm $\left.t\right|_{p}$ of term $t$ at position $p$, and the term $t[s]_{p}$ obtained by replacing $\left.t\right|_{p}$ by $s$ are defined in the standard way. For example, if $t$ is $f(a, g(b, h(c)), d)$, then $\left.t\right|_{2.2 .1}=c$, and $t[d]_{2.2}=f(a, g(b, d), d)$. The empty sequence, denoted by $\lambda$, corresponds to the root position. We denote $t\left[s_{1}\right]_{p_{1}}\left[s_{2}\right]_{p_{2}} \ldots\left[s_{n}\right]_{p_{n}}$ by either $t\left[s_{1}, s_{2}, \ldots, s_{n}\right]_{p_{1}, p_{2}, \ldots, p_{n}}$, or $t\left[s_{1}, \ldots, s_{n}\right]_{P}$, where $P=\left\{p_{1}, \ldots, p_{n}\right\}$. By $\left.t\right|_{P}$ we denote the set $\left\{\left.t\right|_{p_{i}}: p_{i} \in P\right\}$. By $P o s(t)$ we denote the set of all positions $p$ such that $\left.t\right|_{p}$ is defined. We write $p_{1} \succ p_{2}$ (equivalently, $p_{2} \prec p_{1}$ ) and say $p_{1}$ is below $p_{2}$ (equivalently, $p_{2}$ is above $p_{1}$ ) if $p_{2}$ is a proper prefix of $p_{1}$, that is, $p_{1}=p_{2} \cdot p_{2}^{\prime}$ for some nonempty $p_{2}^{\prime}$. Positions $p$ and $q$ are disjoint if $p \nsucceq q$ and $q \nsucceq p$.

We will often denote a term $f\left(t_{1}, \ldots, t_{n}\right)$ by the simplified form $f t_{1} \ldots t_{n}$, and $t[s]_{p}$ by $t[s]$ when $p$ is clear by the context or not important. By $\operatorname{Vars}(t)$ we denote the set of all variables occurring in $t$. The height of a term $s$ is 0 if $s$ is a variable or a constant, and $1+\max _{i} h e i g h t\left(s_{i}\right)$ if $s=f\left(s_{1}, \ldots, s_{m}\right)$. The depth of a position $p$ is the length of $p$. The size of a term $f s_{1} \ldots s_{m}$ is $1+\sum_{i=1}^{m} \operatorname{size}\left(s_{i}\right)$.

A substitution $\sigma$ is sometimes presented explicitly as $\left\{x_{1} \mapsto t_{1}, \ldots, x_{n} \mapsto t_{n}\right\}$. We assume standard definition for a rewrite rule $l \rightarrow r$, a rewrite system $R$, the one step rewrite relation at position $p$ induced by $R \rightarrow_{R, p}$, and the one step rewrite relation induced by $R$ (at any position) $\rightarrow_{R}$. The notations $\leftrightarrow, \rightarrow^{+}$, and $\rightarrow^{*}$, are standard [5].

A rewrite system $R$ is confluent if the relation $\leftarrow_{R}^{*} \circ \rightarrow_{R}^{*}$ is contained in $\rightarrow^{*} \circ \leftarrow^{*}$, which is equivalent to the relation $\leftrightarrow_{R}^{*}$ being contained in $\rightarrow^{*} \circ \leftarrow^{*}$ (called the Church-Rosser property). A term $t$ is reachable from $s$ by $R$ (or, $R$-reachable) if $s \rightarrow_{R}^{*} t$. A set $S$ of terms is said to be equivalent by $R$ (or, $R$-equivalent) if $s \leftrightarrow{ }_{R}^{*} t$ for all $s, t \in S$. A set $S$ of terms is $R$-joinable if there is a term that is $R$-reachable from all of them. A (rewrite) derivation or proof (from $s$ ) is a sequence of rewrite steps (starting from $s$ ), that is, a sequence $s \rightarrow_{R} s_{1} \rightarrow_{R} s_{2} \rightarrow_{R} \ldots$

A term $t$ is called ground if $t$ contains no variables. It is called shallow if all variable positions in $t$ are at depth 0 or 1 . It is called linear if every variable occurs at most once in $t$. It is flat if its height is at most 1 . A rule $l \rightarrow r \in R$ is called shallow right-linear if the term $r$ is linear, and both $l, r$ are shallow; it is flat if both $l, r$ are flat terms. A flat rule $l \rightarrow r$ is called a permutation rule if height $(l)=$ height $(r)=1$; it is called a decreasing rule if height $(l)=1$ and $h e i g h t(r)=0$, and an increasing rule if height $(l)=0$ and height $(r)=1$.

\section{Confluence}

Let $R$ be such that every rule $l \rightarrow r \in R$ is shallow and right-linear. Using standard transformation rules, the rewrite system $R$ can be transformed into a rewrite system $R^{\prime}$ such that every rule $l \rightarrow r \in R^{\prime}$ is flat and right-linear. This transformation is achieved by introducing new constants and adding new rewrite rules $[7,9]$. Additionally, we can also assume that $\Sigma=\Sigma_{0} \cup\{f\}$, where $f$ is of 
arity $m$. All these transformations preserve confluence. We assume henceforth that $R$ is a flat and right-linear $T R S$ defined over a signature $\Sigma=\Sigma_{0} \cup\{f\}$. We want to decide if $R$ is confluent.

We use the fact that $R$-equivalence is decidable $[4,12]$ repeatedly below. Since shallow and right-linear systems are finite-path overlapping and right-linear, the $R$-reachability and $R$-joinability relations are also decidable [13]. We assume that the theory of the rewrite system $R$ is not trivial, that is, it is not the case that $x \leftrightarrow_{R}^{*} y$. Confluence of such systems can be decided by simply checking if $x$ and $y$ are $R$-joinable. We also assume that $R$ contains no rule of the form $x \rightarrow t$ where $x \notin \operatorname{Vars}(t)$. Any $R$ that contains such a rule is trivially confluent.

\subsection{Top-Stabilizable Constants}

A term $t \in \mathcal{T}(\Sigma, \mathcal{V})$ is called top-stable if it cannot be rewritten to a constant in $\Sigma_{0}$ by $R$, that is, there is no constant $c \in \Sigma_{0}$ s.t $t \rightarrow_{R}^{*} c$. A constant $c$ is topstabilizable if it is $R$-equivalent to a top-stable term. Our intention is, for every set $\left\{c_{1}, \ldots, c_{k}\right\}$ of equivalent constants that are top-stabilizable, to choose a new marked representative constant, say $\overline{c_{1}}$, and add the $k$ rules $c_{1} \rightarrow \overline{c_{1}}, c_{2} \rightarrow$ $\overline{c_{1}}, \ldots, c_{k} \rightarrow \overline{c_{1}}$ to $R$. The intuitive idea for adding these rules is that they allow the rewrite system to replace a top-stabilizable constant by an equivalent constant which can not be used by the rewrite system (note that these new constants do not appear in $R$ ).

Let $\bar{\Sigma}_{0}$ be a new set of constants obtained by picking a representative constant from each set of $R$-equivalent constants and marking it.

$\bar{\Sigma}_{0}=\left\{\bar{c}: c \in \Sigma_{0}, c\right.$ is a chosen representative for its $R$-equivalence class $\}$

We next define $\bar{R}$ (over the new signature $\Sigma \cup \bar{\Sigma}_{0}$ ) by adding certain rewrite rules of the form $c \rightarrow \bar{d}$, where $c$ and $d$ are $R$-equivalent. The construction of $\bar{R}$ is achieved through a fixpoint computation.

$$
\begin{aligned}
R_{0} & =R \\
R_{i+1} & =R_{i} \cup\left\{c \rightarrow \bar{d}: c, d \in \Sigma_{0}, \exists \text { flat term } t \in \mathcal{T}\left(\Sigma \cup \bar{\Sigma}_{0}, \mathcal{V}\right): t \leftrightarrow{ }_{R_{i}}^{*} c \leftrightarrow{ }_{R}^{*} d,\right. \\
& \left.\bar{d} \in \bar{\Sigma}_{0}, t \text { does not rewrite to a constant in } \Sigma_{0} \text { by } R_{i}\right\}
\end{aligned}
$$

When we add $c \rightarrow \bar{d}$, we also add all rewrite rules $c^{\prime} \rightarrow \bar{d}$, where $c^{\prime} \leftrightarrow_{R}^{*} c$, in the same iteration, and hence, the fixpoint iterations terminate in at most $\left|\overline{\Sigma_{0}}\right|$ steps. Let $\bar{R}$ be the final result.

Example 1. If $R_{0}=\left\{f a \rightarrow b, a \rightarrow a^{\prime}, f b \rightarrow c\right\}$, then $R_{1}=R_{0} \cup\{b \rightarrow \bar{b}\}$ (due to the witness $f a^{\prime}$ ) and subsequently $R_{2}=R_{1} \cup\{c \rightarrow \bar{c}\}$ (due to the witness $f \bar{b}$ ).

The next lemma states that the addition of the new constants does not change the congruence relation (over the original signature).

Lemma 1. If $s, t \in \mathcal{T}(\Sigma, \mathcal{V})$, then for all $i, s \leftrightarrow_{R_{i}}^{*} t$ iff $s \leftrightarrow_{R}^{*} t$. 
We would want a rule $c \rightarrow \bar{d}$ to be added if and only if the constant $c$ is top-stabilizable. This is not always the case, but the left-to-right implication is stated in Lemma 5. Its proof uses the following definitions and lemmas. These lemmas are not conceptually difficult but they are of technical nature, and they are used again later, in the last part of Lemma 12.

Definition 1. Let $s \rightarrow_{l \rightarrow r, q}$ t be a one-step rewrite derivation with a flat rightlinear rule and let $p \in \operatorname{Pos}(s)$. We say that $p$ goes to a certain position $p^{\prime}$ in this derivation, denoted by Post $(s \rightarrow t)(p)=p^{\prime}$, whenever:

$-p^{\prime}=p$, and either (i) $q$ is disjoint from $p$, or (ii) $p \prec q$, or (iii) $p=q$ and $l$ is not a variable, or

$-p=q \cdot q_{1} \cdot p^{\prime \prime}$ and $p^{\prime}=q \cdot q_{2} \cdot p^{\prime \prime}$, for some $q_{1}, q_{2}$ such that $\left.l\right|_{q_{1}}$ and $\left.r\right|_{q_{2}}$ are the same variable.

If there is no such $p^{\prime}$, then we say that $p$ does not go anywhere, denoted by $\operatorname{Post}(s \rightarrow t)(p)=\perp$.

We extend this definition to derivations of arbitrary length. If $s=s_{1} \rightarrow s_{2} \rightarrow$ $\cdots \rightarrow s_{n}$, we say that $p_{1}$ goes to $p_{n}$, or Post $\left(s \rightarrow^{*} t\right)\left(p_{1}\right)=p_{n}$, if there exists $p_{2}, \ldots, p_{n-1}$ such that for all $i, \operatorname{Post}\left(s_{i} \rightarrow s_{i+1}\right)\left(p_{i}\right)=p_{i+1}$. If no such sequence of positions exists, we say that $p$ does not go anywhere.

For example, in the derivation $f(f a a b) b c \rightarrow_{f x x y \rightarrow f x b c, 1} f(f a b c) b c$, position 1 goes to position $1,1.1$ and 1.2 go to 1.1 , and 1.3 does not go anywhere.

Lemma 2. Let $R^{\prime}$ be any flat right-linear rewrite system and Post $\left(s \rightarrow R_{R^{\prime}}^{*}\right.$ $t)(\lambda)=p$. Then $\left.s \rightarrow_{R^{\prime}}^{*} t\right|_{p^{\prime}}$ for any $p^{\prime} \preceq p$.

The above lemma depends on the right-shallowness of insertion rules in $R$.

Lemma 3. Let $R_{i}$ be one of the rewrite systems appearing in the construction of $\bar{R}$. Let $s \rightarrow_{R_{i}}^{*} t$ be any derivation. Let $p_{1}, \ldots, p_{k}$ be disjoint positions, that are also disjoint with Post $\left(s \rightarrow^{*} t\right)(\lambda)$ whenever it is not $\perp$, and such that every $\left.t\right|_{p_{j}}$ is $R_{i}$-equivalent to some bar constant $\overline{c_{j}}$.

Then $s \rightarrow_{R_{i}}^{*} t\left[\overline{c_{1}}\right]_{p_{1}} \ldots\left[\overline{c_{k}}\right]_{p_{k}}$, where $\lambda$ goes to the same position as before.

Alternatively, if none of the terms $\left.t\right|_{p_{j}}$ is $R_{i}$-equivalent to any constant, then there is a derivation $s \rightarrow{ }_{R_{i}}^{*} t\left[z_{1}\right]_{p_{1}} \ldots\left[z_{k}\right]_{p_{k}}$, where $\lambda$ goes to the same position as before and $z_{1}, \ldots, z_{k}$ are new variables.

Proof. We use induction on the length of the derivation $s \rightarrow_{R_{i}}^{*} t$. For length 0 the result is trivial since there are no positions disjoint with $\lambda$. Hence, let the derivation be of the form $s \rightarrow_{R_{i}}^{*} t^{\prime} \rightarrow_{l \rightarrow r, q} t$.

If $q \succeq p_{j}$ for some $j$, then it is the case that $t^{\prime}\left[\overline{c_{1}}\right]_{p_{1}} \ldots\left[\overline{c_{k}}\right]_{p_{k}} \equiv t\left[\overline{c_{1}}\right]_{p_{1}} \ldots\left[\overline{c_{k}}\right]_{p_{k}}$. Moreover, the $p_{j}$ 's are disjoint with $\operatorname{Post}\left(s \rightarrow_{R_{i}}^{*} t^{\prime}\right)(\lambda)$. By induction hypothesis, $s \rightarrow{ }_{R_{i}}^{*} t^{\prime}\left[\overline{c_{1}}\right]_{p_{1}} \ldots\left[\overline{c_{k}}\right]_{p_{k}}$ with $\lambda$ going to the same position as before.

Now suppose that $q \nsucceq p_{j}$ for any $j$. For every $p_{j}$, define a set of maximal disjoint positions $P_{j}=\operatorname{Pre}\left(t^{\prime} \rightarrow t\right)\left(p_{j}\right) \subseteq \operatorname{Pos}\left(t^{\prime}\right)$ as follows:

$$
\operatorname{Pre}\left(t^{\prime} \rightarrow t\right)\left(p_{j}\right)=\operatorname{Maximal}\left(\left\{p \in \operatorname{Pos}\left(t^{\prime}\right): \operatorname{Post}\left(t^{\prime} \rightarrow t\right)(p)=p_{j}\right\}\right),
$$


where $\operatorname{Maximal}(P)$ denotes the set of maximal positions in $P$ (wrt $\succ$ ). Now, note that every $P_{j}$ is a set of disjoint positions, but moreover, all of them are disjoint with $\operatorname{Post}\left(s \rightarrow_{R_{i}}^{*} t^{\prime}\right)(\lambda)$, and $\cup_{j \in\{1 \ldots k\}} P_{j}$ is a set of disjoint positions. Hence, by induction hypothesis, $s \rightarrow{ }_{R_{i}}^{*} t^{\prime}\left[\overline{c_{1}}, \ldots, \overline{c_{1}}\right]_{P_{1}} \ldots\left[\overline{c_{k}}, \ldots, \overline{c_{k}}\right]_{P_{k}}$ with $\lambda$ going to the same place, and $t^{\prime}\left[\overline{c_{1}}, \ldots, \overline{c_{1}}\right]_{P_{1}} \ldots\left[\overline{c_{k}}, \ldots, \overline{c_{k}}\right]_{P_{k}} \rightarrow t^{\prime \prime}$, where $t^{\prime \prime}$ may differ from $t\left[\overline{c_{1}}\right]_{p_{1}} \ldots\left[\overline{c_{k}}\right]_{p_{k}}$ in the positions $p_{j}$ of the form $q . l$ for some $l \in\{1, \ldots, m\}$ such that $\left.r\right|_{l}$ is a constant. For such a position $p_{j},\left.\left.\left.t^{\prime \prime}\right|_{p_{j}} \equiv t\right|_{p_{j}} \equiv r\right|_{l}$, and hence, this constant is $R_{i}$-equivalent to the corresponding $\overline{c_{j}}$. Therefore, by applying rules of the form $c \rightarrow \overline{c_{j}}$ on $t^{\prime \prime}$, the term $t\left[\overline{c_{1}}\right]_{p_{1}} \ldots\left[\overline{c_{k}}\right]_{p_{k}}$ is reached, and hence, this term is also $R_{i}$-reachable from the initial $s$, and with $\lambda$ going to the same position. The proof for the alternate claim follows the same pattern.

Example 2. Let $S=\{x \rightarrow g x c, c \rightarrow f c\}, R=R_{0} \cup S$ and $\bar{R}=R_{2} \cup S$, where $R_{0}$ and $R_{2}$ are as in Example 1. In the $\bar{R}$-derivation $b \rightarrow g b c \rightarrow g(g b c) c \rightarrow g(g b c) f c$, the position $\lambda$ goes to position 1.1. We can replace $c$ and $f c$ in the disjoint positions 1.2 and 2 by $\bar{c}$ and get a new derivation $b \rightarrow g b c \rightarrow g(g b c) c \rightarrow g(g b \bar{c}) \bar{c}$.

We can replace top-stable subterms by equivalent bar-constants in certain $R_{i^{-}}$ derivations (Lemma 4). Recall that a rewrite step using $l \rightarrow r$ is called decreasing if height $(l)=1$ and height $(r)=0$.

Lemma 4. Let $R_{i}$ be one of the rewrite systems appearing in the construction of $\bar{R}$ and let $f s_{1} \ldots s_{m}$ be a flat term over $\Sigma \cup \overline{\Sigma_{0}}$. Let $f s_{1}^{\prime} \ldots s_{m}^{\prime}$ be a term obtained from $f s_{1} \ldots s_{m}$ by replacing every bar constant by an $R_{i}$-equivalent and $R$-top-stable term in $\mathcal{T}(\Sigma, \mathcal{V})$. Let $f s_{1}^{\prime} \ldots s_{m}^{\prime} \rightarrow_{R}^{*} t\left[f t_{1}^{\prime} \ldots t_{m}^{\prime}\right]_{p}$ be a derivation in which $\lambda$ goes to $p$, and that does not have any decreasing steps applied at the positions where $\lambda$ goes to.

Then, there exists a derivation $f s_{1} \ldots s_{m} \rightarrow_{R_{i}}^{*} t\left[f t_{1} \ldots t_{m}\right]_{p}$, where $\lambda$ goes to $p$, and $f t_{1} \ldots t_{m}$ is obtained from $f t_{1}^{\prime} \ldots t_{m}^{\prime}$ by replacing every $R$-top-stable $t_{j}^{\prime}$ $R_{i}$-equivalent to a bar constant $t_{j}$ by this corresponding $t_{j}$, and leaving the other $t_{j}^{\prime}$ unchanged, that is, $t_{j}=t_{j}^{\prime}$.

Proof. We induct on the length of the derivation $f s_{1}^{\prime} \ldots s_{m}^{\prime} \rightarrow_{R}^{*} t\left[f t_{1}^{\prime} \ldots t_{m}^{\prime}\right]_{p}$. For length 0 the result is trivial since in this case $t$ is the empty context and every $t_{j}^{\prime}$ coincides with the corresponding $s_{j}^{\prime}$. Hence, let this derivation be of the form $f s_{1}^{\prime} \ldots s_{m}^{\prime} \rightarrow_{R}^{*} t^{\prime} \rightarrow_{l \rightarrow r \in R, q} t\left[f t_{1}^{\prime} \ldots t_{m}^{\prime}\right]_{p}$. Now, we distinguish several cases depending on the relationship between $q$ and $p$.

$q$ and $p$ are disjoint. In this case $\operatorname{Post}\left(f s_{1}^{\prime} \ldots s_{m}^{\prime} \rightarrow_{R_{i}}^{*} t^{\prime}\right)(\lambda)=p$ and $t^{\prime}$ is actually $t^{\prime}\left[f t_{1}^{\prime} \ldots t_{m}^{\prime}\right]_{p}$. By induction hypothesis, $f s_{1} \ldots s_{m} \rightarrow_{R_{i}}^{*} t^{\prime}\left[f t_{1} \ldots t_{m}\right]_{p}$ in which $\lambda$ goes to $p$. Moreover, $t^{\prime}\left[f t_{1} \ldots t_{m}\right]_{p} \rightarrow_{l \rightarrow r, q} t\left[f t_{1} \ldots t_{m}\right]_{p}$, and hence $t\left[f t_{1} \ldots t_{m}\right]_{p}$ is $R_{i}$-reachable from $f s_{1} \ldots s_{m}$, with $\lambda$ going to $p$.

$q \prec p$, or $q=p$ and $\operatorname{Post}\left(f s_{1}^{\prime} \ldots s_{m}^{\prime} \rightarrow_{R_{i}}^{*} t^{\prime}\right)(\lambda) \neq q$. (In the latter case, the step $t^{\prime} \rightarrow_{l \rightarrow r \in R_{i}, q} \quad t\left[f t_{1}^{\prime} \ldots t_{m}^{\prime}\right]_{p}$ is necessarily a decreasing step.) Let $P=\operatorname{Pre}\left(t^{\prime} \rightarrow t[\ldots]_{p}\right)(p)$. Note that there is a position $p^{\prime} \in P$ such that Post $\left(f s_{1}^{\prime} \ldots s_{m}^{\prime} \rightarrow^{*} t^{\prime}\right)(\lambda)=p^{\prime}$. By induction hypothesis, $f s_{1} \ldots s_{m} \rightarrow_{R_{i}}^{*} t^{\prime}\left[f t_{1} \ldots t_{m}\right]_{p^{\prime}}$. Using Lemma 3 on this derivation at positions below the other positions in $P$, we get $f s_{1} \ldots s_{m} \rightarrow_{R_{i}}^{*} t^{\prime \prime}$, where 
$t^{\prime \prime}$ is $t^{\prime}\left[f t_{1} \ldots t_{m}, \ldots, f t_{1} \ldots t_{m}\right]_{P}$. We have set up $t^{\prime \prime}$ so that $t^{\prime \prime} \rightarrow_{l \rightarrow r, q}$ $t\left[f t_{1} \ldots t_{m}\right]_{p}$, and hence $t\left[f t_{1} \ldots t_{m}\right]_{p}$ is $R_{i}$-reachable from $f s_{1} \ldots s_{m}$, with $\lambda$ going to $p$.

$q=p$ and $\operatorname{Post}\left(f s_{1}^{\prime} \ldots s_{m}^{\prime} \rightarrow_{R_{i}}^{*} t^{\prime}\right)(\lambda)=q$. In this case, $l \rightarrow r$ has to be a permutation rule: by assumption, no decreasing rules occur at the positions where $\lambda$ goes to, and an increasing step would imply $\operatorname{Post}\left(f s_{1}^{\prime} \ldots s_{m}^{\prime} \rightarrow{ }_{R_{i}}^{*}\right.$ $\left.t\left[f t_{1}^{\prime} \ldots t_{m}^{\prime}\right]_{p}\right)(\lambda) \neq p$. Therefore, $t^{\prime}$ is of the form $t\left[f r_{1}^{\prime} \ldots r_{m}^{\prime}\right]_{p}$ and $f r_{1}^{\prime} \ldots r_{m}^{\prime}$ rewrites to $f t_{1}^{\prime} \ldots t_{m}^{\prime}$ by $l \rightarrow r$. By induction hypothesis, $f s_{1} \ldots s_{m} \rightarrow{ }_{R_{i}}^{*}$ $t\left[f r_{1} \ldots r_{m}\right]_{p}$, where $\lambda$ goes to $p$. Note that the same permutation rule $l \rightarrow r$ is applicable at $\lambda$ in $f r_{1} \ldots r_{m}$, since $r_{j}^{\prime} \equiv r_{k}^{\prime}$ implies $r_{j} \equiv r_{k}$ for all $j, k$. Hence, $f s_{1} \ldots s_{m} \rightarrow{ }_{R_{i}}^{*} t\left[f t_{1} \ldots t_{m}\right]_{p}$, where $\lambda$ goes to $p$ in this derivation.

$p \prec q$. In this case, $t^{\prime}$ is of the form $t\left[f t_{1}^{\prime} \ldots t_{j-1}^{\prime} r_{j}^{\prime} t_{j+1}^{\prime} \ldots t_{m}^{\prime}\right]_{p}$, where $r_{j}^{\prime} \rightarrow_{l \rightarrow r} t_{j}^{\prime}$. Note that either $t_{j} \equiv t_{j}^{\prime}$ or $t_{j}$ is a bar-constant $R_{i}$-equivalent to $t_{j}^{\prime}$. In either case, $t_{j}$ is $R_{i}$-equivalent to $r_{j}^{\prime}$.

If $t_{j} \equiv t_{j}^{\prime}$, then $r_{j}^{\prime}$ is not an $R$-top-stable term that is $R_{i}$-equivalent to a bar constant occurring in $R_{i}$. By induction hypothesis there is a derivation $f s_{1} \ldots s_{m} \rightarrow_{R_{i}}^{*} t\left[f t_{1} \ldots t_{j-1} r_{j}^{\prime} t_{j+1} \ldots t_{m}\right]_{p}$, where $\lambda$ goes to $p$. Since $r_{j}^{\prime} \rightarrow t_{j}^{\prime}$, it follows that $f s_{1} \ldots s_{m} \rightarrow_{R_{i}}^{*} t\left[f t_{1} \ldots t_{m}\right]_{p}$ with $\lambda$ going to $p$.

If $t_{j}$ is a bar-constant $R_{i}$-equivalent to $t_{j}^{\prime}$, then $t_{j}^{\prime}$ is $R$-top-stable. If $r_{j}^{\prime}$ is also $R$-top-stable, then induction hypothesis gives exactly what we wanted to prove. Otherwise, if $r_{j}^{\prime}$ is not $R$-top-stable, then, induction hypothesis gives a derivation $f s_{1} \ldots s_{m} \rightarrow_{R_{i}}^{*} t\left[f t_{1} \ldots t_{j-1} r_{j}^{\prime} t_{j+1} \ldots t_{m}\right]_{p}$, where $\lambda$ goes to $p$. Moreover, since $r_{j}^{\prime}$ is not $R$-top-stable, we have $r_{j}^{\prime} \rightarrow_{R}^{*} c$ for some constant $c \in \Sigma$, which is $R_{i}$-equivalent to the bar-constant $t_{j}$, and hence, the rule $c \rightarrow t_{j}$ occurs in $R_{i}$. Therefore, $t\left[f t_{1} \ldots t_{m}\right]_{p}$ is $R_{i}$-reachable from $f s_{1} \ldots s_{m}$, with $\lambda$ going to $p$.

Example 3. Using $\bar{R}$ from Example 2, in the derivation $g\left(f a^{\prime}\right) c \rightarrow g\left(f a^{\prime}\right) f c \rightarrow$ $g\left(g f a^{\prime} c\right) f c, \lambda$ goes to $\lambda$, and by Lemma 4 we would have a derivation $g \bar{b} c \rightarrow^{*} g \bar{b} \bar{c}$.

Lemma 5. If there is a rule $c \rightarrow \bar{d}$ in $\bar{R}$, then $d$ is $R$-top-stabilizable.

Proof. We prove by induction on $i$ that if $c \rightarrow \bar{d}$ is introduced in $R_{i}$, then $d$ is $R$-top-stabilizable. Suppose this is not true for certain $i$ and $c \rightarrow \bar{d}$; i.e., $d$ is not top-stabilizable but this rule has been introduced in the $i$ 'th step. By the construction of $R_{i}$, there exists a flat term $s=f s_{1} \ldots s_{m}$ in $\mathcal{T}\left(\Sigma \cup \bar{\Sigma}_{0}, \mathcal{V}\right) R_{i-1^{-}}$ equivalent to $d$ and such that $s$ does not rewrite to any constant in $\Sigma_{0}$ by $R_{i-1}$. We can assume that any $s_{j}$ that is a bar constant already occurs in $R_{i-1}$ : a bar constant $s_{j}$ not occurring in $R_{i-1}$ can be replaced by a new variable, preserving all properties and the proof. We construct a term $s^{\prime}=f s_{1}^{\prime} \ldots s_{m}^{\prime}$ as follows: if $s_{j}$ is some bar constant $\bar{e}$ then $s_{j}^{\prime}$ is chosen as an $R$-top-stable term in $\mathcal{T}(\Sigma, \mathcal{V})$ $R$-equivalent to $e$ that exists by induction hypothesis, and if $s_{j}$ is not a bar constant then we make $s_{j}^{\prime}$ equal to $s_{j}$. By Lemma $1, s^{\prime}$ and $d$ are $R$-equivalent. Since $d$ is not $R$-top-stabilizable, $s^{\prime} \rightarrow_{R}^{*} c^{\prime}$ for some $c^{\prime}$ equivalent to $d$. Wlog, assume that all terms that occur in the derivation $s^{\prime} \rightarrow_{R}^{*} c^{\prime}$ are in $\mathcal{T}(\Sigma, \mathcal{V})$.

First, suppose that there is a decreasing step at some position where $\lambda$ goes to in the derivation $s^{\prime} \rightarrow_{R}^{*} c^{\prime}$. Hence, this derivation can be written as 
$f s_{1}^{\prime} \ldots s_{m}^{\prime} \rightarrow_{R}^{*} t\left[f t_{1}^{\prime} \ldots t_{m}^{\prime}\right]_{p} \rightarrow_{R, p} t^{\prime} \rightarrow_{R}^{*} c^{\prime}$, where the step $t\left[f t_{1}^{\prime} \ldots t_{m}^{\prime}\right]_{p} \rightarrow_{R, p} t^{\prime}$ is the first decreasing step at the positions where $\lambda$ goes to. By Lemma 4 , there exists a derivation $f s_{1} \ldots s_{m} \rightarrow_{R_{i-1}}^{*} t\left[f t_{1} \ldots t_{m}\right]_{p}$ where $\lambda$ goes to $p$, and $f t_{1} \ldots t_{m}$ is obtained from $f t_{1}^{\prime} \ldots t_{m}^{\prime}$ by replacing every $R$-top-stable $t_{j}^{\prime}$ that is

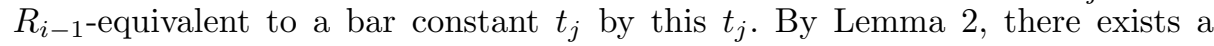
derivation $f s_{1} \ldots s_{m} \rightarrow{ }_{R_{i-1}}^{*} f t_{1} \ldots t_{m}$. Since $R$ is flat, the decreasing step applied on the subterm $f t_{1}^{\prime} \ldots t_{m}^{\prime}$ can be also applied on $f t_{1} \ldots t_{m}$ and the result is either a constant or one of the $t_{j}$ 's. The first case is not possible since then $f s_{1} \ldots s_{m}$ would $R_{i-1}$-reach a constant, which is a contradiction. In the second case, we have $f s_{1} \ldots s_{m} \rightarrow{ }_{R_{i-1}}^{*} t_{j}$. Note that $t_{j}$ can not be one of the introduced bar constants, since they are not equivalent to $d$. Hence, $t_{j} \in \mathcal{T}(\Sigma, \mathcal{V})$ and $t_{j}$ is $R$-equivalent to $d$. Since $d$ is not $R$-top-stabilizable, there is a derivation $t_{j} \rightarrow{ }_{R}^{*} c^{\prime \prime}$ for some constant equivalent to $d$. But this derivation is also an $R_{i-1}$-derivation since $R \subseteq R_{i-1}$, and hence, $f s_{1} \ldots s_{m} R_{i-1}$-reaches a constant, which is a contradiction.

We can now assume that there are no decreasing steps at the positions where $\lambda$ goes to in $s^{\prime} \rightarrow_{R}^{*} c^{\prime}$. This implies, in particular, that $\lambda$ does not go anywhere in this derivation. Hence, the derivation is of the form $f s_{1}^{\prime} \ldots s_{m}^{\prime} \rightarrow_{R}^{*}$ $t\left[f t_{1}^{\prime} \ldots t_{m}^{\prime}\right]_{p} \rightarrow_{l \rightarrow r \in R, p^{\prime}} t^{\prime} \rightarrow_{R}^{*} c^{\prime}$, where $\lambda$ goes to $p$, and there exists $p^{\prime \prime} \in$ $\operatorname{Pos}(l)$ such that $\left.l\right|_{p^{\prime \prime}}$ is a variable not occurring in $r$, and $p^{\prime} \cdot p^{\prime \prime} \preceq p$. As before, by Lemma 4 , there exists a derivation $f s_{1} \ldots s_{m} \rightarrow_{R_{i-1}}^{*} t\left[f t_{1} \ldots t_{m}\right]_{p}$ where $\lambda$ goes to $p$. From $t=t\left[f t_{1} \ldots t_{m}\right]_{p}$ we construct a new term $t^{\prime \prime}=$ $t\left[\left.t\right|_{p^{\prime} \cdot p^{\prime \prime}}\right]_{p^{\prime} \cdot p_{1}} \ldots\left[\left.t\right|_{p^{\prime} \cdot p^{\prime \prime}}\right]_{p^{\prime} \cdot p_{k}}$, where $p_{1} \ldots p_{k}$ are all the positions in $l$ where the variable $\left.l\right|_{p^{\prime \prime}}$ occurs. By Lemma $3, f s_{1} \ldots s_{m} \rightarrow_{R_{i-1}}^{*} t^{\prime \prime}$. By construction of $t^{\prime \prime}$, $t^{\prime \prime} \rightarrow_{l \rightarrow r \in R, p^{\prime}} t^{\prime} \rightarrow_{R}^{*} c^{\prime}$, and since $R \subseteq R_{i-1}$, it follows that $f s_{1} \ldots s_{m} R_{i-1^{-}}$ reaches a constant, which contradicts the assumption on $f s_{1} \ldots s_{m}$.

\subsection{Detection of Top-Stabilizable Constants}

Not all the top-stabilizable constants are necessarily detected by the fix-point computation. But under certain confluence assumptions, we can guarantee that some of them will be detected.

Lemma 6. Let $R$ be confluent upto height h, i.e., any set of equivalent terms with height smaller than or equal to $h$ is joinable.

Then, if $t$ is a top-stable term with height smaller than or equal to $h+1$ and equivalent to some constant $c$, then $c \rightarrow \bar{d} \in \bar{R}$ for some $\bar{d} \in \overline{\Sigma_{0}}$.

For proving the previous lemma, we first need some properties about the congruence relation induced by $R$. Since $R$ is a shallow TRS, $R$-equivalence can be decided using a paramodulation-based completion procedure $[4,12]$. The resulting saturated TRS can be used as the set $\operatorname{Congr}(R)$, or alternatively, we can just use the following:

$$
\operatorname{Congr}(R)=\left\{l \rightarrow r: l, r \text { are flat, } l \leftrightarrow_{R}^{*} r, \text { height }(l) \geq h \text { eight }(r)\right\} .
$$

This set could have nonlinear terms on the right-hand sides. In this section, we study some properties of rewriting with a flat TRS $R$. 
Definition 2. Let $t$ be a term. A set of disjoint positions $P \neq\{\lambda\}$ of $t$ is called maximally equivalent if all $\left.t\right|_{p \in P}$ are equivalent, and for any $t_{q}$ equivalent to them and different from $t$ there exists some $p \in P$ with $p \preceq q$. If a term $s$ (or a set of terms $S$ ) is equivalent to $\left.\right|_{P}$, we say that $P$ is the maximally equivalent set in $t$ equivalent to $s(S)$.

The following lemma shows that, in some cases, the relation $\rightarrow{ }_{R}^{*}$ is preserved after replacing some subterms by variables.

Lemma 7. Let $R$ be a flat TRS. Let $s \rightarrow_{\text {Congr }(R)}^{*} t$, and let $P$ be maximally equivalent in $s$ such that the terms $\left.s\right|_{p \in P}$ are not equivalent to a constant.

Then, either $s[z, \ldots, z]_{P} \rightarrow_{\operatorname{Congr}(R)}^{*} z$ and $s$ is equivalent $\left.s\right|_{P}$, or $s[z, \ldots, z]_{P} \rightarrow_{\text {Congr }(R)}^{*} t[z, \ldots, z]_{P^{\prime}}$, where $P^{\prime}$ is the maximally equivalent set in $t$ equivalent to the terms $\left.s\right|_{p \in P}$.

Proof. We prove it for one step derivations, since the proof inductively extends to any length. Hence, assume that $s \rightarrow_{\operatorname{Congr}(R), q} t$ and $P$ is as above.

If $p \preceq q$ for some $p \in P$, then $\left.s\right|_{p^{\prime}}$ and $\left.t\right|_{p^{\prime}}$ are equivalent for any $p^{\prime} \preceq p$ and for any $p^{\prime}$ disjoint with $p$, and the result trivially follows. Hence, assume that $p \npreceq q$ for any $p \in P$. Note that $\left.s\right|_{p^{\prime}}$ and $\left.t\right|_{p^{\prime}}$ are equivalent for any $p^{\prime} \preceq q$ and for any $p^{\prime}$ disjoint with $q$.

If the applied rule is of the form $f(\ldots, x, \ldots) \rightarrow x$ with the $x$ of the left-hand side occurring in a position $i$ such that $q . i \in P$, then $\left.t\right|_{q}$ is equivalent to terms $\left.s\right|_{P}$. In such a case, $\left.s\right|_{q}$ is also equivalent to $\left.s\right|_{P}$, and since $q$ was not in $P$, it can only be that $q=\lambda$ and $s[z, \ldots, z]_{P} \rightarrow \operatorname{Congr}(R) z$ trivially.

In any other case, the maximally equivalent set in $t$ equivalent to $\left.s\right|_{P}$ is the set $\left\{p^{\prime} \mid p^{\prime} \in P\right.$ disjoint with $\left.q\right\} \cup\left\{q \cdot p^{\prime} \mid p^{\prime}\right.$ is in the maximally equivalent set of $\left.t\right|_{q}$ equivalent to $\left.\left.s\right|_{P}\right\}$. Let $l \rightarrow r$ and $\sigma$ be the applied rule and substitution. Let $\sigma^{\prime}$ be as $\sigma$ except for the variables $x \in \operatorname{Vars}(r)-\operatorname{Vars}(l)$, for which we define $x \sigma^{\prime}=x \sigma[z, \ldots, z]_{P_{x}}$, where $P_{x}=\{\lambda\}$ if $x \sigma$ is equivalent to $\left.s\right|_{P}$, and $P_{x}$ is the maximally equivalent set in $x \sigma$ equivalent to $\left.s\right|_{P}$, otherwise. Then, $s[z, \ldots, z]_{P}$ rewrites into $t[z, \ldots, z]_{P^{\prime}}$ applying $l \rightarrow r$ with $\sigma^{\prime}$ at position $q$ (note that positions with constants in $r$ are not equivalent to $\left.s\right|_{P}$ since, by the assumptions of the lemma, $\left.s\right|_{P}$ is not equivalent to a constant).

Corollary 1. Let $s_{1}, \ldots, s_{n}$ be terms that reach a term $t$ by $\rightarrow_{\text {Congr }(R)}^{*}$. Let $P_{1}, \ldots, P_{n}$ be maximally equivalent positions in $s_{1}, \ldots, s_{n}$, respectively, such that all terms in $\left.s_{1}\right|_{P_{1}}, \ldots,\left.s_{n}\right|_{P_{n}}$ are equivalent, but not equivalent to a constant.

Then, either some $s_{i}[z, \ldots, z]_{P_{i}}$ reaches $z$ by $\rightarrow_{\text {Congr }(R)}^{*}$ and the $s_{j}$ 's are all equivalent to $\left.s_{i}\right|_{P_{i}}$, or all $s_{i}[z, \ldots, z]_{P_{i}}$ 's reach the same term $t[z \ldots z]_{P^{\prime}}$ by $\operatorname{Congr}(R)$, where $P^{\prime}$ is the maximal set in $t$ equivalent to $\left.s_{i}\right|_{P_{i}}$; and hence, they are all equivalent.

Let $R$ and $\bar{R}$ be as in the previous subsection. The following lemma shows that, in some cases, an $\bar{R}$-derivation can be transformed into an $R$-one. 
Lemma 8. For every bar constant $\bar{c}$, let $t_{\bar{c}}$ be a term $R$-reachable from all constants equivalent to $c$. Let $s$ and $t$ be two terms satisfying $s \rightarrow \frac{*}{R} t$.

Then, $s\left\{\ldots \bar{c} \mapsto t_{\bar{c}} \ldots\right\} \rightarrow_{R}^{*} t\left\{\ldots \bar{c} \mapsto t_{\bar{c}} \ldots\right\}$.

Proof. It is enough to prove it for one step derivations, since then it inductively extends to any length. Hence, assume $s \rightarrow_{\bar{R}} t$. If this step uses a rule in $R$, then the result is trivial. Otherwise, it uses a rule of the form $d \rightarrow \bar{c}$, and the result trivially follows from the fact that $t_{\bar{c}}$ is reachable from $d$.

\section{Now, we are ready to prove Lemma 6}

Proof. (of Lemma 6) The proof is by contradiction. We consider a term $t$ as a counterexample witness to the goal, if its height is smaller than or equal to $h+1$, $t$ is top-stable, and it is equivalent to a constant that has not been detected as top-stabilizable. We compare witnesses by the size ordering.

Assume that the minimal counterexample witness is a certain term $t$ equivalent to some constant $c$.

First, we show that all terms occurring in $t$ at depth 1 and with non-zero height are equivalent to constants. Suppose not. Let $s$ be a height non-zero subterm of $t$ at depth 1 that is not equivalent to a constant. Let $P$ be the maximally equivalent set of positions of $t$ equivalent to $s$. Since $t \rightarrow_{\operatorname{Congr}(R)}^{*} c$, by Lemma 7 , either $t[z, \ldots, z]_{P} \rightarrow_{\text {Congr }(R)}^{*} z$ and $t$ is equivalent to $s$, or $t[z, \ldots, z]_{P} \rightarrow_{\operatorname{Congr}(R)}^{*} c[z, \ldots, z]_{P^{\prime}}$, where $P^{\prime}$ is the maximally equivalent set in $c$ equivalent to $s$. The first case is not possible, since $s$ is not equivalent to a constant and $t$ is equivalent to $c$. In the second case, $c[z, \ldots, z]_{P^{\prime}}=c$, and hence $t[z, \ldots, z]_{P}$ is a term equivalent to $c$ and smaller than $t$. Therefore, by the minimality assumptions on $t$, the term $t[z, \ldots, z]_{P}$ is not stable, i.e. $t[z, \ldots, z]_{P} \rightarrow_{R}^{*} d$ for some constant $d$ equivalent to $c$. On the other hand, the set $\left.t\right|_{P}$ is equivalent and all its terms have height smaller than $h+1$, and hence it is joinable to some term $r$. Consequently $t$ reaches $t[r, \ldots, r]_{P}$, and the derivation $\left(t[z, \ldots, z]_{P} \rightarrow_{R}^{*} d\right)\{z \mapsto r\}$ shows that $t[r, \ldots, r]_{P}$ reaches $d$, which contradicts the fact that $t$ is top-stable.

Furthermore, by minimality of $t$ it follows that every such height non-zero subterm $s$ at depth 1 in $t$ is top-stable, and moreover, its corresponding equivalent constant has been detected as top-stabilizable, since otherwise, we would have a smaller counterexample witness $\{s\}$.

Now, let $t^{\prime}$ be like $t$ but where every height non-zero depth 1 subterm is replaced by its corresponding $\bar{R}$-equivalent bar-constant. Clearly, $t^{\prime}$ is flat and $t^{\prime} \leftrightarrow \frac{*}{R} c$. Since $c$ was not detected as top-stabilizable, $t^{\prime} \bar{R}$-rewrites to some constant $d$ equivalent to $c$. For every bar-constant $\bar{e}$, let $S_{\bar{e}}$ be the set of all constants $R$-equivalent to $e$, plus all the terms equivalent to $e$ occurring at depth 1 in $t$. Each such $S_{\bar{e}}$ is joinable, since the height of its terms is smaller than $h+1$, and hence, we can choose a term $t_{\bar{e}}$ reachable from $S_{\bar{e}}$. By Lemma $8, t^{\prime}\{\ldots \bar{e} \mapsto$ $\left.t_{\bar{e}} \ldots\right\} \rightarrow_{R}^{*} d\left\{\ldots \bar{e} \mapsto t_{\bar{e}} \ldots\right\}$. But $d\left\{\ldots \bar{e} \mapsto t_{\bar{e}} \ldots\right\}$ is $d$ and $t^{\prime}\left\{\ldots \bar{e} \mapsto t_{\bar{e}} \ldots\right\}$ is reachable from $t$, and this contradicts the fact that $t$ is top-stable. 


\subsection{Deciding Confluence for Shallow Right-Linear Systems}

Before proving the decidability of confluence, we need some additional lemmas that show that rewrite derivations using shallow and right-linear rules can be generalized to yield "more-general" rewrite derivations.

Lemma 9. Let $s$ be a flat term such that every constant in $s$ also occurs in $\bar{R}$. Let $s \rightarrow \frac{*}{R} t[r]_{p}$ be a derivation where $\lambda$ goes to $p$, and without decreasing steps applied at the positions where $\lambda$ goes to. Let $r^{\prime}$ be $r[z]_{i}$ where $i \in\{1, \ldots, m\}$ and either $\left.r\right|_{i}$ is equivalent to the variable $z$, or $\left.r\right|_{i}$ is not equivalent to any height 0 term and $z$ is a new variable.

Then, there exists a derivation $s \rightarrow \frac{*}{R} t\left[r^{\prime}\right]_{p}$, where $\lambda$ goes to $p$.

The proof of the previous lemma, generalized to several positions $i_{1}, \ldots, i_{k}$, is completely analogous to the one of Lemma 4.

Lemma 10. Suppose $s \rightarrow \frac{*}{R} t$ and Post $\left(s \rightarrow^{*} t\right)(\lambda)=p \neq \perp$. Let $\alpha$ be a barconstant $\bar{R}$-equivalent to $s$ if such a bar-constant exists, and let $\alpha$ be a variable if $s$ is not $\bar{R}$-equivalent to any constant.

Then, for all $p^{\prime} \preceq p$, there is a derivation $\alpha \rightarrow \frac{*}{R} t[\alpha]_{p^{\prime}}$ in which $\lambda$ goes to the position $p^{\prime}$.

Proof. We prove by induction on the length of the derivation $s \rightarrow \frac{*}{R} t$. The base case is trivial. Assume the above derivation is of the form $s \rightarrow \frac{*}{R} t[l \sigma]_{q} \rightarrow t[r \sigma]_{q}$, and take $p^{\prime} \preceq p$. We analyze the following cases:

(a) If $p$ and $q$ are disjoint, then $\lambda$ goes to $p$ in $s \rightarrow^{*} t[l \sigma]_{q}$. Applying induction hypothesis on this derivation we have $s \rightarrow^{*} t[l \sigma]_{q}[\alpha]_{p^{\prime}}$ with $\lambda$ going to $p^{\prime}$, and the same rule $l \rightarrow r$ applied at position $q$ finishes the proof.

(b) If $p^{\prime} \preceq q$ then $\lambda$ goes to a position below or at $p^{\prime}$ in $s \rightarrow \frac{*}{R} t[l \sigma]$, and induction hypothesis on this derivation establishes the claim.

(c) Suppose $p=q \cdot i \cdot q^{\prime}$ and $p^{\prime}=q \cdot i \cdot q^{\prime \prime}$. We again distinguish two cases. If $l$ is a variable, then $\lambda$ goes to $q . q^{\prime}$ in $s \rightarrow^{*} t[l \sigma]$. By induction hypothesis, we get $\alpha \rightarrow^{*} t[\alpha]_{q \cdot q^{\prime \prime}}$. Now, apply the rule $l \rightarrow r$ at position $q$ to rewrite $t[\alpha]_{q \cdot q^{\prime \prime}}$ into $t[\alpha]_{q . i . q^{\prime \prime}}$. This concludes the first case. In the second case, we assume that $l$ is not a variable. Apply induction hypothesis on the subderivation $s \rightarrow^{*} t[l \sigma]_{q}$ at the appropriate position in $P=\operatorname{Pre}(t[l \sigma] \rightarrow t[r \sigma])\left(p^{\prime}\right)$. We replace the terms at other positions in $P$ by $\alpha$ using Lemma 3 to finally get the derivation $\alpha \rightarrow \frac{*}{R}$ $\left(t[l \sigma]_{q}\right)[\alpha, \ldots, \alpha]_{P}$. Note that this is required because $l$ may be nonlinear. Now, we can use the rule $l \rightarrow r$ to rewrite $\left(t[l \sigma]_{q}\right)[\alpha, \ldots, \alpha]_{P}$ into $\left(t[r \sigma]_{q}\right)[\alpha]_{p^{\prime}}$.

Example 4. Applying Lemma 10 to the derivation $b \rightarrow^{*} g(g b c) f c$ of Example 2, we infer that there will be derivations $\bar{b} \rightarrow^{*} g(g \bar{b} c) f c, \bar{b} \rightarrow^{*} g \bar{b} f c$, and $\bar{b} \rightarrow^{*} \bar{b}$.

The following lemma is an addendum to Lemma 4.

Lemma 11. Let $s$ be a flat term with constants occurring in $\bar{R}$, and let $s^{\prime}$ be obtained by replacing in $s$ every bar-constant by an $\bar{R}$-equivalent $R$-top-stable term in $\mathcal{T}(\Sigma, \mathcal{V})$. Let $s^{\prime} \rightarrow_{R}^{*} t$ be a derivation where $\lambda$ does not go anywhere, and without decreasing steps applied at the positions where $\lambda$ goes to. 
Then, there exists a derivation $s \rightarrow \frac{*}{R} t$, where $\lambda$ does not go anywhere. Moreover, if $s$ is not $\bar{R}$-equivalent to a constant, then there exists a derivation $z \rightarrow \frac{*}{R} t$ for any variable $z$, and where $\lambda$ does not go anywhere.

We are ready to give a characterization for confluence of $R$.

Lemma 12. $R$ is confluent iff the following two conditions hold:

(i) Every R-equivalent set of constants of $\Sigma$ is R-joinable.

(ii) Let $\left\{\alpha_{1}, \ldots, \alpha_{k}, t_{1}, \ldots, t_{n}\right\}$ be an $\bar{R}$-equivalent set of terms, where $n \geq 1$, $k+n \geq 2, \alpha_{i}$ 's are constants in $\Sigma_{0}$ or variables (in this case there is at most one variable, i.e. $k \in\{0,1\}$ ), and $t_{i}$ 's are flat terms over $\Sigma \cup \bar{\Sigma}_{0}$ such that no $t_{i}$ can reach a constant in $\Sigma$ or a variable by $\rightarrow \frac{*}{R}$.

Then, there exist $t_{1}^{\prime}, \ldots, t_{n}^{\prime}$ such that every $t_{i}^{\prime}$ is either $t_{i}$ or $\bar{c}$ or $x$, some $t_{i}^{\prime}$ coincides with $t_{i}$, and the set $\left\{\alpha_{1}, \ldots, \alpha_{k}, t_{1}^{\prime}, \ldots, t_{n}^{\prime}\right\}$ is $\bar{R}$-joinable. Here $\bar{c}$ is the (possible) bar-constant in the equivalence class of the set and $x$ is the (possible) variable in the equivalence class of the set.

Proof. $\Leftarrow$ : For the right-to-left direction, we will prove a more general statement: $R$ is confluent, and all top-stabilizable constants have been detected (during the fixpoint computation that constructs $\bar{R}$ ), i.e., $\bar{d}$ occurs in $\bar{R}$ if $\bar{d} \in \overline{\Sigma_{0}}$ and $d$ is top-stabilizable. The proof is by contradiction, and we consider two kinds of counterexamples to the goal: a multiset $\left\{t_{1}, \ldots, t_{n}\right\}$ with $n \geq 2$ is a counterexample witness to confluence if it is equivalent but not joinable. A single set $\{t\}$ is a witness to the top-stabilizability detection if $t$ is top-stable but it is equivalent to a constant that has not been detected as top-stabilizable. We compare witnesses $\left\{t_{1}, \ldots, t_{n}\right\}$ using the multiset extension of the size ordering.

Witness to top-stabilizability detection: Assume that the minimal counterexample witness is $\{t\}$, where $t$ is top-stable and equivalent to some constant $c$ that has not been detected as top-stabilizable.

By minimality of $\{t\}$, all equivalent sets of terms with height smaller than the height of $t$ are joinable. Therefore, by Lemma 6 , we conclude that $c$ has been detected as top-stabilizable, which is a contradiction.

Witness to confluence: Assume that the minimal counterexample witness is a witness to confluence. The witness can not contain only constants, due to condition (i). Let $\left\{\alpha_{1}, \ldots, \alpha_{k}, t_{1}, \ldots, t_{n}\right\}$ be the minimal counterexample witness to confluence, where the $t_{i}$ 's are not constants, $n \geq 1$ and $n+k \geq 2$.

We first prove that all terms occurring in the $t_{i}$ 's at depth 1 and with height non-zero are equivalent to constants. Suppose not. Let $s$ be a height nonzero subterm at depth 1 of some term $t_{i}$ such that $s$ is not equivalent to a constant. We pick a term $t$ reachable from all $\left\{t_{1}, \ldots, t_{n}\right\}$ by $\rightarrow_{\operatorname{Congr}(R)}$. If there are constants in the witness $(k \geq 1)$, we choose $t$ to be a constant. Let $P_{1}, \ldots, P_{n}$ be the maximal positions equivalent to $s$ in $t_{1}, \ldots, t_{n}$, respectively. By Corollary 1 , either for some $t_{i}$, say $t_{1}$, it happens that $t_{1}[z \ldots z]_{P_{1}} \rightarrow_{\operatorname{Congr}(R)} z$ and $t_{1}$ is equivalent to $\left.t_{1}\right|_{P_{1}}$ and to $s$ (in this case no constant appears in the witness, i.e., $k=0$ ), or all the $t_{i}[z, \ldots, z]_{P_{i}}$ 's reach the same term $t[z \ldots z]_{P^{\prime}}$ by $\operatorname{Congr}(R)$, where $P^{\prime}$ is maximal in $t$, and hence they are all equivalent (in this case, if there 
are constants in the witness, $P^{\prime}$ is empty and $t[z \ldots z]_{P^{\prime}}$ is exactly the constant $t)$.

In the first case, $\left\{t_{1}[z, \ldots, z]_{P_{1}}, z\right\}$ and $\left.t_{1}\right|_{P_{1}} \cup\left\{t_{2}, \ldots, t_{n}\right\}$ are both equivalent sets smaller than the original witness. Therefore, each of these two sets is separately joinable, say to terms $u$ and $v$ respectively. Instantiating $z$ by $v$ in $z \rightarrow^{*} u$, we get $v \rightarrow^{*} u\{z \mapsto v\}$. As a result we infer that $u\{z \mapsto v\}$ is reachable from every term in $\left\{t_{1}, \ldots, t_{n}\right\}$, contradicting that this set is a counterexample to confluence.

In the second case, $\left\{\alpha_{1}, \ldots, \alpha_{k}, t_{1}[z \ldots z]_{P_{1}}, \ldots, t_{n}[z \ldots z]_{P_{n}}\right\}$ and $\left.t_{1}\right|_{P_{1}} \cup \ldots \cup$ $\left.t_{n}\right|_{P_{n}}$ are both $R$-equivalent sets that are smaller than the original witness. Therefore, both these sets are separately $R$-joinable, say to terms $u$ and $v$ respectively. It is again easy to see that any term in $\left\{\alpha_{1}, \ldots, \alpha_{k}, t_{1}, \ldots, t_{n}\right\}$ reaches $u\{z \mapsto v\}$, contradicting the fact this set is a counterexample for confluence.

We now know that in the minimal counterexample $\left\{\alpha_{1}, \ldots, \alpha_{k}, t_{1}, \ldots, t_{n}\right\}$, any height non-zero term $s$ occurring at depth 1 is equivalent to a constant $c$. By the minimality of the counterexample, $s$ is top-stable, and hence the constant $c$ is top-stabilizable. Moreover, this has been detected, since $\{s\}$ is smaller than the above witness, and therefore, a bar-constant for the class of $c$ exists. Let $t_{1}^{\prime}, \ldots, t_{n}^{\prime}$ be as $t_{1}, \ldots, t_{n}$, but where every nonzero term occurring at depth 1 has been replaced by its $\bar{R}$-equivalent bar-constant.

First, we show that no $t_{i}^{\prime}$ can reach a constant of $\Sigma$. Suppose that some $t_{i}^{\prime}$, say $t_{1}^{\prime}$, reaches a constant $c \in \Sigma$. For every bar-constant $\bar{d}$, let $S_{\bar{d}}$ be the set of all constants $R$-equivalent to $d$, plus all the terms equivalent to $d$ occurring at depth 1 in $t_{1}$. Each such $S_{\bar{d}}$ is joinable, since it is a smaller set compared to the original witness. Hence, we can choose a term $t_{\bar{d}}$ reachable from $S_{\bar{d}}$. By Lemma $8, t_{1}^{\prime}\left\{\ldots \bar{d} \mapsto t_{\bar{d}} \ldots\right\} \rightarrow_{R}^{*} c$, and since $t_{1}$ reaches $t_{1}^{\prime}\left\{\ldots \bar{d} \mapsto t_{\bar{d}} \ldots\right\}$ by $\rightarrow_{R}^{*}$, it follows that $t_{1}$ reaches a constant, contradicting that $t_{1}$ is top-stable (which follows from minimality of the counterexample).

Now, by Condition (ii), there exist terms $t_{1}^{\prime \prime}, \ldots, t_{n}^{\prime \prime}$ such that every $t_{i}^{\prime \prime}$ is either $t_{i}^{\prime}$ or the corresponding bar-constant of its class (if it is equivalent to a constant), some $t_{i}^{\prime \prime}$ coincides with $t_{i}^{\prime}$, and the set $\left\{\alpha_{1}, \ldots, \alpha_{k}, t_{1}^{\prime \prime}, \ldots, t_{n}^{\prime \prime}\right\}$ is $\bar{R}$-joinable to a certain term $r$. For every bar-constant $\bar{c}$, let $S_{\bar{c}}$ be now the set of all constants $R$ equivalent to $c$, plus all the terms equivalent to $c$ occurring at depth 1 in the $t_{i}$ 's such that $t_{i}^{\prime \prime}$ is not a bar-constant, plus all the terms $t_{i}$ equivalent to $c$ such that $t_{i}^{\prime \prime}$ is a bar-constant. Each such $S_{\bar{c}}$ is joinable, since it is a smaller set than the initial witness, and hence, we can choose a term $t_{\bar{c}}$ reachable from $S_{\bar{c}}$. By Lemma 8 , every term in $\left\{\alpha_{1}, \ldots, \alpha_{k}, t_{1}^{\prime \prime}, \ldots, t_{n}^{\prime \prime}\right\}\left\{\ldots \bar{c} \mapsto t_{\bar{c}} \ldots\right\}$ reaches $r\left\{\ldots \bar{c} \mapsto t_{\bar{c}} \ldots\right\}$. Since every $t_{i}$ reaches $t_{i}^{\prime \prime}\left\{\ldots \bar{c} \mapsto t_{\bar{c}} \ldots\right\}$, this proves that $\left\{\alpha_{1}, \ldots, \alpha_{k}, t_{1}, \ldots, t_{n}\right\}$ is joinable, which contradicts that it is a witness to confluence.

$\Rightarrow$ : Assume that $R$ is confluent. This immediately implies Condition (i). Moreover, by Lemma 6 , all the top-stabilizable constants have been detected. To show that Condition (ii) is also true, let $\left\{\alpha_{1}, \ldots, \alpha_{k}, t_{1}, \ldots, t_{n}\right\}$ be a set as in Condition (ii).

We choose terms $t_{1}^{\prime}, \ldots, t_{n}^{\prime}$ such that every $t_{i}^{\prime}$ can be obtained by replacing in $t_{i}$ every bar-constant at depth 1 by an $\bar{R}$-equivalent $R$-top-stable term of the 
original signature. The set $\left\{\alpha_{1}, \ldots, \alpha_{k}, t_{1}^{\prime}, \ldots, t_{n}^{\prime}\right\}$ is $\bar{R}$-equivalent and contains terms of the original signature, and by Lemma 1 it is also $R$-equivalent. By confluence, it is $R$-joinable to a certain term $t$, i.e. there exist derivations of the form $\alpha_{i} \rightarrow_{R}^{*} t$ and $t_{i}^{\prime} \rightarrow_{R}^{*} t$. We choose such a $t$ to be a minimal one in size satisfying such condition. Note that, due to this minimality, every height nonzero subterm of $t$ is top-stable.

Let $t_{i}$, say $t_{1}$, be such that a decreasing step occurs in the derivation $t_{1}^{\prime} \rightarrow_{R}^{*} t$ at some position where $\lambda$ goes to. Let $t_{1}^{\prime} \rightarrow_{R}^{*} s\left[r_{1}^{\prime}\right]_{p} \rightarrow s\left[r_{2}^{\prime}\right]_{p}$ be the initial subderivation where a decreasing step appears for the first time. Note that $\lambda$ goes to $p$ in this derivation, no decreasing step occurs at the positions where $\lambda$ goes to in $t_{1}^{\prime} \rightarrow_{R}^{*} s\left[r_{1}^{\prime}\right]_{p}$, and $r_{1}^{\prime}$ rewrites to $r_{2}^{\prime}$ with a decreasing step at root position. By Lemmas 4 and $9, t_{1} \rightarrow \frac{*}{R} s\left[r_{1}\right]_{p}$ where $\lambda$ goes to $p$, and $r_{1}$ is as $r_{1}^{\prime}$ but where, fixing a new variable $z$, every height nonzero subterm at depth 1 has been replaced by, either $z$ if it is not equivalent to any height 0 term, or by an equivalent bar-constant if it is equivalent to some constant, or by an equivalent variable if it is equivalent to a variable. The same decreasing rule used in $r_{1}^{\prime} \rightarrow_{R} r_{2}^{\prime}$ can be applied to $r_{1}$ obtaining a certain term $r_{2}$ that is either a bar-constant, or a variable. Therefore, $t_{1} \rightarrow \frac{*}{R} s\left[r_{2}\right]_{p}$ where $\lambda$ goes to $p$, and by Lemma $2 t_{1} \rightarrow \frac{*}{R} r_{2}$. Therefore, $\left\{\alpha_{1}, \ldots, \alpha_{k}, t_{1}, r_{2}, \ldots, r_{2}\right\}$ is $\bar{R}$-joinable and we are done.

At this point, we can assume that no derivation $t_{i}^{\prime} \rightarrow_{R}^{*} t$ contains a decreasing step at some position where $\lambda$ goes to, and we distinguish two cases.

The $t_{i}^{\prime}$ 's are not equivalent to any height 0 term. In this case $k=0$.

First we show that in every derivation $t_{i}^{\prime} \rightarrow_{R}^{*} t, \lambda$ goes to somewhere. Suppose not, i.e. wlog. assume that $\lambda$ does not go anywhere in $t_{1}^{\prime} \rightarrow_{R}^{*} t$. By Lemma 11 there exists a derivation $z \rightarrow \frac{*}{R} t$ for any variable $z$. We choose $z$ to be a new variable not occurring in $t$. This shows that the theory induced by $R$ is trivial, which contradicts the initial assumptions of this section.

Next we show that the positions where $\lambda$ goes to in the derivations $t_{i}^{\prime} \rightarrow_{R}^{*} t$ are not disjoint. Suppose not, i.e. wlog. assume that $\lambda$ goes to disjoint $p_{1}$ and $p_{2}$ in $t_{1}^{\prime} \rightarrow_{R}^{*} t$ and $t_{2}^{\prime} \rightarrow_{R}^{*} t$, respectively. Using Lemma 10 on these two derivations, we get derivations $x \rightarrow \frac{*}{R} t[x]_{p_{1}}$ and $y \rightarrow \frac{*}{R} t[y]_{p_{2}}$. Now, using Lemma 3 on these, we get derivations $x \rightarrow \frac{*}{R} t[x]_{p_{1}}[y]_{p_{2}}$ and $y \rightarrow \frac{*}{R} t[y]_{p_{2}}[x]_{p_{1}}$. This shows that $x$ and $y$ are $\bar{R}$-equivalent, and by Lemma $1 R$-equivalent, which contradicts again the initial assumption of the non-triviality of the theory induced by $R$.

Finally we show that the positions where $\lambda$ goes to in the derivations $t_{i}^{\prime} \rightarrow_{R}^{*} t$ coincide. Suppose not, i.e. wlog. assume that $\lambda$ goes to $p_{1}$ and $p_{1} . i . p_{2}$ in $t_{1}^{\prime} \rightarrow_{R}^{*} t$ and $t_{2}^{\prime} \rightarrow_{R}^{*} t$, respectively. By Lemma 4 applied to $t_{1}^{\prime} \rightarrow_{R}^{*} t$, we get $t_{1} \rightarrow \frac{*}{R} t[r]_{p_{1}}$. Note that, by Lemma 2 applied to $t_{2}^{\prime} \rightarrow_{R}^{*} t, t_{2}^{\prime}$ is $R$-equivalent to $\left.t\right|_{p_{1} . i}$, and hence $\left.t\right|_{p_{1} . i}$ is not $R$-equivalent to a height 0 term, and $\left.t\right|_{p_{1} . i}$ and $\left.r\right|_{i}$ coincide. Therefore, using Lemma 9 we get $t_{1} \rightarrow \frac{*}{R} t\left[r[y]_{i}\right]_{p_{1}}$. On the other hand, by Lemma 3 , applied to $t_{2}^{\prime} \rightarrow_{R}^{*} t$ we get $t_{2} \rightarrow \bar{R} t[r]_{p_{1}}$. Using Lemma 10 on this derivation, we get $y \rightarrow \frac{*}{R} t\left[r[y]_{i}\right]_{p_{1}}$. This shows that an arbitrary variable $y$ is $\bar{R}$-equivalent to $t_{1}$, contradicting the non-triviality of the theory induced by $R$. 
Once we know that there exists a position $p$ where $\lambda$ goes to in all of the derivations $t_{i}^{\prime} \rightarrow_{R}^{*} t$, it is easy to conclude: by Lemma 4 any $t_{i} \bar{R}$-reaches $t[r]_{p}$, where $r$ is obtained from $\left.t\right|_{p}$ by replacing every height nonzero subterm at depth $1 R$-equivalent to a constant by a bar-constant, and hence $\left\{t_{1}, \ldots, t_{n}\right\}$ is $\bar{R}$ joinable.

The set $\left\{\alpha_{1}, \ldots, \alpha_{k}, t_{1}, \ldots, t_{n}\right\}$ is $\bar{R}$-equivalent to a height 0 term. If $\lambda$ does not go anywhere in a certain derivation $t_{i}^{\prime} \rightarrow_{R}^{*} t$, by Lemma 11, $t_{i} \rightarrow \frac{*}{R} t$. Therefore, if $\lambda$ does not go anywhere in any derivation $t_{i}^{\prime} \rightarrow_{R}^{*} t$, then $\left\{\alpha_{1}, \ldots, \alpha_{k}, t_{1}, \ldots, t_{n}\right\}$ is $\bar{R}$-joinable to $t$ and we are done. Hence, from now on we assume that $\lambda$ goes to somewhere in some of the derivations $t_{i}^{\prime} \rightarrow_{R}^{*} t$, say in $t_{1}^{\prime} \rightarrow_{R}^{*} t, \ldots, t_{l}^{\prime} \rightarrow_{R}^{*} t$, for some $l \geq 1$ and $l \leq n$, and let $p_{1}, \ldots, p_{l}$ the positions where $\lambda$ goes to in each of these derivations, respectively. From the list of positions $p_{1}, \ldots, p_{l}$ we are interested in the ones that are minimal. Wlog. assume that, for some $o, p_{1}, \ldots, p_{o}$ are the minimal ones, i.e., for every $i$ in $\{o+1, \ldots, l\}$ there exists a $j$ in $\{1, \ldots, o\}$ such that $p_{j} \prec p_{i}$. Now, we define the term $t^{\prime}$ by fixing a new variable $z$, and replacing in $t$ every height nonzero subterm at position $p_{i} . j$, for $i$ in $\{1, \ldots, o\}$ and $j$ in $\{1, \ldots, m\}$, by either an equivalent barconstant if it is $R$-equivalent to a constant, or by an equivalent variable if it is $R$-equivalent to a variable, or by $z$ if it is not $R$-equivalent to a height 0 term.

Let $\alpha$ be the height 0 term $\bar{R}$-equivalent to $\left\{\alpha_{1}, \ldots, \alpha_{k}, t_{1}, \ldots, t_{n}\right\}$. The term $\alpha$ can be a bar-constant or variable. We finish the proof by showing that $t^{\prime}$ is $\bar{R}$-reachable from all terms in $\left\{\alpha_{1}, \ldots, \alpha_{k}, t_{1}, \ldots t_{o}, \alpha, \ldots, \alpha, t_{l+1}, \ldots, t_{n}\right\}$ (the $t_{i}$ 's for $i$ in $\{o+1, \ldots, l\}$ are replaced by $\alpha$ ).

- For a term $t_{i}$ with $i$ in $\{1, \ldots, o\}$, this follows from Lemmas 4,9 and 3 .

- For a term $t_{i}$ with $i$ in $\{l+1, \ldots, n\}$, this follows from Lemmas 11 and 3 .

- For a term $t_{i}$ with $i$ in $\{o+1, \ldots, l\}, \lambda$ goes to a certain $p_{j} \cdot j^{\prime} \cdot p_{i}^{\prime}$ in the derivation $t_{i} \rightarrow_{R}^{*} t$ for some $j$ in $\{1, \ldots, o\}$ and $j^{\prime}$ in $\{1, \ldots, m\}$. By Lemma 10, there is a derivation $\alpha \rightarrow \frac{*}{R} t[\alpha]_{p_{j} \cdot j^{\prime}}$, where $\lambda$ goes to $p_{j} \cdot j^{\prime}$, and now, the fact that $\alpha \rightarrow \frac{*}{R} t^{\prime}$ follows from Lemma 3 .

- For a term $\alpha_{i}$ such that $\lambda$ does not go anywhere in $\alpha_{i} \rightarrow_{R}^{*} t$ or it goes to a position $p$ disjoint with $p_{1}, \ldots, p_{o}$, this follows from Lemma 3 .

- For a term $\alpha_{i}$ such that $\lambda$ goes to a position $p_{j} \cdot j^{\prime} \cdot p$ for some $i$ in $\{1, \ldots, o\}$ and $j^{\prime}$ in $\{1, \ldots, m\}$, from Lemma 10 it follows that $\alpha \rightarrow \frac{*}{R} t[\alpha]_{p_{j} . j^{\prime}}$, where $\lambda$ goes to $p_{j} \cdot j^{\prime}$. If $\alpha$ is a variable then it coincides with $\alpha_{i}$, and if $\alpha$ is a bar constant then $\alpha_{i} \rightarrow \alpha$ is a rule in $\bar{R}$. In either case there is a derivation $\alpha_{i} \rightarrow \frac{*}{R} t[\alpha]_{p_{j} \cdot j^{\prime}}$ where $\lambda$ goes to $p_{j} \cdot j^{\prime}$. Now, the fact that $\alpha_{i} \rightarrow \frac{*}{R} t^{\prime}$ follows from Lemma 3.

Theorem 1. Confluence of shallow right-linear rewrite systems is decidable.

Proof. Since a shallow and right-linear system $R$ is finite-path overlapping and right-linear, $R$-reachability and $R$-joinability are decidable [13]. $R$-equivalence is decidable for shallow rewrite systems $[4,12]$. As a result, the set $\bar{R}$ can be constructed and the conditions of Lemma 12 can be tested. 


\section{Conclusion}

In this paper, we showed that confluence is decidable for shallow right-linear rewrite systems, thus generalizing the result for shallow linear rewrite systems [8]. The new proof uses the decidability results for reachability and joinability [13] and the word problem $[4,12]$. We also prove many properties about rewriting using shallow TRSs and also shallow right-linear TRSs, which are used to prove the main results of this paper. The decidability of termination and confluence for other classes of finite-path overlapping systems is left for future investigation.

\section{References}

1. L. Bachmair and H. Ganzinger. Rewrite-based equational theorem proving with selection and simplification. J. of Logic and Computation, 4:217-247, 1994.

2. A. Bouajjani. Languages, rewriting systems, and verification of infinite-state systems. In ICALP, volume 2076 of $L N C S$, pages 24-39. Springer, 2001.

3. H. Comon, M. Dauchet, R. Gilleron, F. Jacquemard, D. Lugiez, S. Tison, and M. Tommasi. Tree automata techniques and applications. Available on: http://www.grappa.univ-lille3.fr/tata, 1997.

4. H. Comon, M. Haberstrau, and J.-P. Jouannaud. Syntacticness, cyclesyntacticness, and shallow theories. Information and Computation, 111(1):154-191, 1994.

5. N. Dershowitz and J. P. Jouannaud. Rewrite systems. In J. van Leeuwen, editor, Handbook of Theoretical Computer Science (Vol. B: Formal Models and Semantics), pages 243-320, Amsterdam, 1990. North-Holland.

6. J. Giesl and H. Zantema. Liveness in rewriting. In RTA, volume 2706 of $L N C S$, pages 321-336. Springer, 2003.

7. G. Godoy and A. Tiwari. Deciding fundamental properties of right-(ground or variable) rewrite systems by rewrite closure. In Intl. Joint Conf. on Automated Deduction, IJCAR, volume 3097 of LNAI, pages 91-106, 2004.

8. G. Godoy, A. Tiwari, and R. Verma. On the confluence of linear shallow term rewrite systems. In 20th Intl. Symp. on Theor. Aspects of Comp. Sci. STACS 2003, volume 2607 of $L N C S$, pages 85-96. Springer, 2003.

9. G. Godoy, A. Tiwari, and R. Verma. Deciding confluence of certain term rewriting systems in polynomial time. Annals of Pure and Applied Logic, 130(1-3):33-59, Dec 2004.

10. F. Jacquemard. Reachability and confluence are undecidable for flat term rewriting systems. Inf. Process. Lett., 87(5):265-270, 2003.

11. N. Martí-Oliet and J. Meseguer. Rewriting logic: roadmap and bibliography. Theor. Comput. Sci., 285(2):121-154, 2002.

12. R. Nieuwenhuis. Basic paramodulation and decidable theories. In Proc. 11th IEEE Symp. on Logic In Comp. Sc. LICS, pages 473-483. IEEE Computer Society, 1996.

13. T. Takai, Y. Kaji, and H. Seki. Right-linear finite path overlapping term rewriting systems effectively preserve recognizability. In Rewriting Techniques and Applications, RTA, volume 1833 of $L N C S$, pages 246-260, 2000. 